\title{
Toledo Crystallization Box: Microgravity Crystallization of Perdeuterated S. typhimurium Tryptophan Synthase for Neutron Diffraction
}

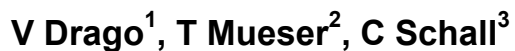 \\ ${ }^{1}$ University of Toledo, Columbiana, $\mathrm{OH},{ }^{2}$ Dept of Chemistry and Biochemistry, Univ. Of Toledo, \\ ${ }^{3}$ University of Toledo, Toledo, $\mathrm{OH}$ \\ victoria.drago@rockets.utoledo.edu
}

As ubiquitous proteins with significant metabolic functions, PLP-dependent enzymes are attractive targets for specific inhibitor design. Tryptophan synthase (TS) is a model for -elimination catalyzed by a PLP-dependent enzyme. In TS, an indole is coupled to a PLP- activated serine, forming tryptophan. Because it lacks a human or animal homolog, TS is an attractive target for inhibition of pathogenic organisms, such as S. aureus. Neutron diffraction of TS will provide positions of hydrogens or deuteriums in the structure, not only allowing for a complete visualization of the active site electronic state but also providing more accurate input models for high order computation. We designed the Toledo Crystallization Box (TCB) to perform capillary dialysis crystallization of perdeuterated TS in microgravity. Our design allows for modulation of equilibration times and sampling of a large variation in crystallization conditions while being highly cost effective. Here, we report the development and employment of the TCB for CASIS PCG 12 and 15 aboard the International Space Station (ISS) for 1 month and 6 months duration, respectively. Preliminary neutron diffraction of perdeuterated TS crystals from PCG 15 exhibited improved neutron diffraction resolution compared to ground control experiments.

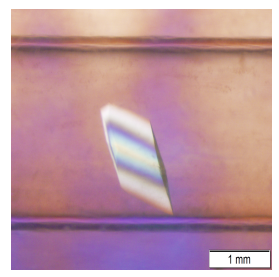

Figure 1

Acta Cryst. (2020). A76, a135 